\title{
PARALLEL LATTICE FILTERS UTILIZING ALUMINUM NITRIDE CONTOUR MODE RESONATORS
}

\author{
K. E. Wojciechowski ${ }^{*}$ and R. H. Olsson III
}

Sandia National Laboratories, Albuquerque, NM, USA

\begin{abstract}
In this work we describe a new parallel lattice (PL) filter topology for electrically coupled AIN microresonator based filters. While $4^{\text {th }}$ order, narrow percent bandwidth $(0.03 \%)$ parallel filters based on high impedance $(11 \mathrm{k} \Omega$ ) resonators have been previously demonstrated at $20 \mathrm{MHz}$ [1], in this work we realize low insertion loss PL filters at 400-500 MHz with termination impedances from 50 to $150 \Omega$ and much wider percent bandwidths, up to $5.3 \%$. Obtaining high percent bandwidth is a major challenge in microresonator based filters given the relatively low piezoelectric coupling coefficients, $k_{t}^{2}$, when compared to bulk (BAW) and surface (SAW) acoustic wave filter materials.
\end{abstract}

\section{INTRODUCTION}

Wide percent $(>1 \%)$ bandwidth filters are needed to achieve the high data rates in applications ranging from CDMA base station transceivers to WiMAX and GSM. These require large percent bandwidth at intermediate frequencies or IF frequencies ( 70-900 MHz). Typically wide bandwidths are achieved at low IF frequencies with SAW devices using Lithium Niobate, $\mathrm{LiNbO}_{3}$, or Lithium Tantalate, $\mathrm{LiTaO}_{3}$ as the filter material. These materials are used because of their high $\mathrm{k}_{\mathrm{t}}^{2}$ allowing for low insertion loss (IL). Unfortunately they are not easily integrated with CMOS or other semiconductor technologies. In addition, SAW and BAW technologies result in large devices at these frequencies for a low motional impedance. The motional impedance per unit area, of a SAW/BAW device, is dependent on frequency due to the direct relationship between wavelength, $\lambda$, of the acoustic wave and electrode spacing. As a result transduction efficiency is dependent on the wavelength, and as $\lambda$ increases the effective transduction coefficient decreases for SAW and BAW devices. This results in large devices at IF frequencies which have significant insertion loss $(-20 \mathrm{~dB})$ in the pass band [2]. It should be noted that BAW or film bulk acoustic wave devices (FBARs) at low IF frequencies are difficult to manufacture and have high insertion loss because they require film thickness that are on the order of 10's of microns (wavelength/2 or $\lambda / 2$ ).

Aluminum Nitride (AIN) contour mode resonators transduced with the $d_{31}$ piezoelectric coefficient have a motional impedance (per unit area) that is independent of device frequency as the acoustic wave in the material is orthogonal to the electric field used to induce it [3]. Hence this form of transduction (dependent on AIN film thickness not wavelength) can result in much smaller filters with similar or lower insertion loss (across a wide range of IF frequencies) than can be achieved with SAW and BAW resonators of equal size.

While SAW filters can create high order filter responses with a single device (advantage SAW), multiple AlN resonators (similar to BAW) must be employed to achieve similar performance. For instance high order ladder or lattice filters can be implemented using multiple resonators. For filter topologies consisting of individual resonators (ladder, lattice) the achievable percent bandwidth of the filter is directly proportional to the separation of the series, $f_{s}$, and parallel resonances, $f_{p}$, of the resonators used in the filter [4]. For BAW resonators this is directly related to the $k_{t}^{2}$ of the material. This is a direct consequence of the transduction mechanism used in these devices. i.e. the resulting capacitance, $C_{o}$, of the piezoelectric transducer is in parallel with the mechanical resonance. For these devices $k_{t}^{2}$ is proportional to the ratio of $C_{x}$, the resonator motional capacitance to $C_{o}$. Or equivalently it is proportional to the ratio ( at resonance $\omega=\omega_{s}=2 \pi f_{s}$ ), of the impedance $Z_{o}=1 /\left(\omega C_{o}\right)$, to the motional resistance of the resonator, $R_{x}$.

$$
\begin{gathered}
k_{t}{ }^{2} \approx \frac{d_{i j}{ }^{2}}{\varepsilon_{i} S_{j j}} \propto \frac{C_{x}}{C_{o}} \propto \frac{Z_{o}}{R_{x}} \\
f_{p}=f_{s} \sqrt{1+\frac{C_{x}}{C_{f t}}}, \text { for } B A W C_{f t}=C_{o}
\end{gathered}
$$

Where $d_{i j}$ is the piezoelectric coefficient, $\varepsilon_{i}$ is the effective permittivity of the piezoelectric material in the $i$ direction and $S_{j j}$ is the mechanical compliance. It is well known, (2), that the separation of the $f_{p}$ and $f_{s}$ is related to the ratio of $C_{x} / C_{o}$ in BAW devices. This is because the feed through or the capacitance in parallel with the mechanical resonator, $C_{f t}$, is equal to $C_{o}$. Hence material properties dictate this separation.

In AIN contour mode resonators use of the $d_{31}$ coefficient enables the decoupling of the relationship between material property $k_{t}^{2}$ and the separation between the series and parallel resonance.

a) Top only transduction (TOT). $w_{e}=$ electrode width, and $\mathrm{S}_{\mathrm{e}}=$ electrode spacing.

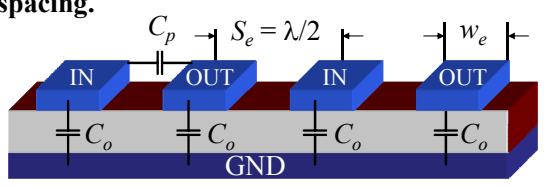

Top electrode Bottom electrode 7 Piezoelectric Film (AIN)

b) Electrical model for TOT.

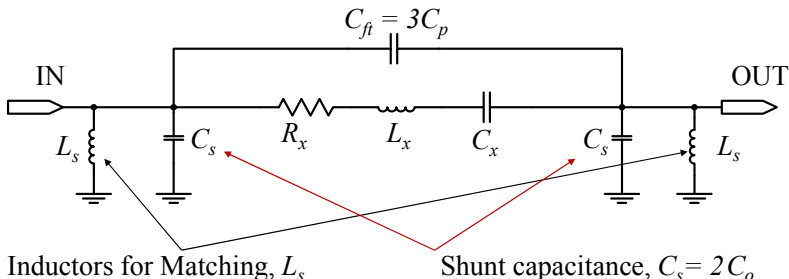

Figure 1: a) Cross section of a TOT AlN overtone resonator. b) electrical equivalent model.

This is achieved by: 1) Using top only transduction (TOT) by arranging the drive and sense electrodes such that the resulting transduction capacitance, $C_{o}$, is a shunt capacitance to ground at the inputs and outputs of the resonator (figure 1a). Therefore reducing the capacitance, $C_{f t}$, which is in parallel with the mechanical resonance. This increases the separation of the series and parallel resonance. 2) The shunt capacitance, $C_{s}$, can be resonated out with matching networks consisting of integrated or off-chip inductors (figure 1b). This recovers insertion loss resulting from this configuration. Conversely, capacitance in 
parallel with the mechanical resonance, $C_{f t}$, cannot be easily removed with matching networks, especially without compromising the low frequency filter rejection.

To summarize the main advantages of AlN contour mode resonators based filters at IF frequencies are: 1) Frequency independent motional impedance which enables small size compared to SAW and FBAR for a given IL. 2) Increased separation between series and parallel resonance enabling wide percent bandwidth filters to be implemented exceeding the material limit set by $k_{t}^{2}$ (Maximum bandwidth $=1.2 \%$ for a $\mathrm{k}_{\mathrm{t}}^{2}=2.4 \%$ in our case) [5]. 3) Multiple resonators with closely spaced center frequencies, required to design wide bandwidth filters, can be realized with good accuracy using AIN contour mode resonators. These advantages enable a new parallel lattice filter topology which can be used to achieve high percentage bandwidth filters at IF frequencies.

\section{PARALLEL LATTICE FILTERS}

a) Basic lattice filter.

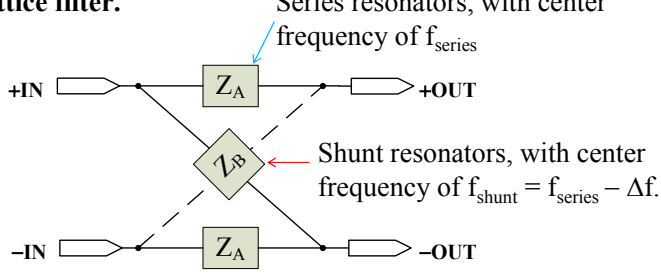

b) Lattice filter implemented with an inverting element. A transformer in this case.

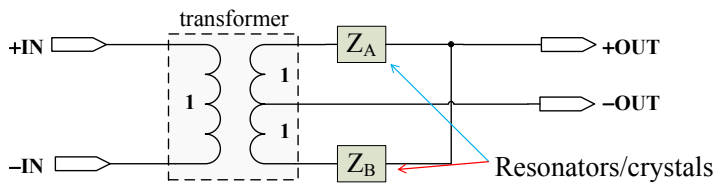

Figure 2: a) Basic lattice filter with series and shunt resonators with impedances, $Z A$ and $Z B$ respectively. b) Alternative implementation of a lattice [4]. Note that this is equivalent to an $L$ $1 / 2$ section in a ladder filter [4].

As was mentioned above, PL filter topology is particularly well suited for implementation with AlN contour mode resonators as they provide key technological advantages which enable parallelization of lattice networks: small feed through capacitance (large separation between $f_{s}$ and $f_{p}$ ), multiple frequency resonators on a single chip, and low impedance resonators [3]. In addition, AlN contour mode resonators provide the ability to perform signal inversion. This removes the need for external inversion required to implement a lattice filter. Finally the PL lattice topology reduces overall filter insertion loss by placing resonators in parallel instead placing them in series as is done with ladder filters.

Consider the basic lattice filter in figure 2a. Alternatively this filter can be implemented using two resonators with the addition of an inversion. In figure $2 \mathrm{~b}$ a transformer (external to the resonators) is added to provide signal inversion [4]. The lattice in figure 2a has also been implemented using active circuitry to provide the inversion [1]. The lattice filters in this work implement a lattice utilizing topologies in figure $1 \mathrm{a}, 3 \mathrm{a}$ and $3 \mathrm{~b}$. Note that the lattices are implemented with electrically coupled resonators and the resonators provide the necessary inversions, hence there is no need for active circuitry. The basic lattice filter shown in figure $2 \mathrm{a}$ has a lower and upper limit on its achievable percent bandwidth. This limit is set by resonator $\mathrm{Q}$ or $3 \mathrm{~dB}$ bandwidth, desired termination impedance, and desired insertion loss. As the series and shunt frequencies are moved closer together the range of frequency where the output responses of the two resonators add constructively shrinks. As a result a narrow pass band can be achieved at the cost of insertion loss.

a) Fully differential lattice using 2 AIN fully differential resonators.

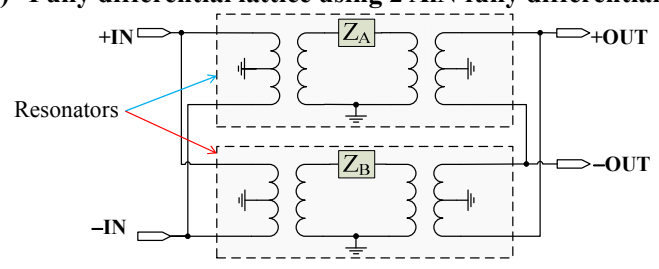

b) Balun lattice filter using $2 \mathrm{AIN}$ balun resonators.

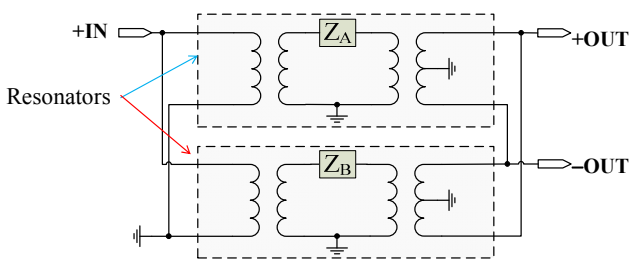

Figure 3: Schematics of electrically coupled AlN resonator based lattice filters. a) Fully differential and b) Balun lattice filters.

For narrow bandwidths increasing resonator area may not be sufficient to overcome the loss. Conversely, as the series and shunt resonances in a lattice are moved apart pass band ripple becomes large and the termination impedances required to flatten the filter response may become unacceptably large. As a result the $3 \mathrm{~dB}$ bandwidth, $f_{3 d B}$, of the resonators, sets a practical upper limit on filter bandwidth $\left(\mathrm{BW}_{3 \mathrm{~dB}}=2 \times \mathrm{f}_{3 \mathrm{~dB}}\right)$ for a two frequency (single lattice) filter (figures $2 \mathrm{a}, \mathrm{b}$ and $3 \mathrm{a}, \mathrm{b}$ ). Note this limit is approximate and depends on the termination impedance requirements as well. Larger bandwidths can be achieved with a single lattice as long as resonator impedance can be made small enough (increase resonator size) to meet ripple and IL specifications for a given termination. Essentially the $3 \mathrm{~dB}$ bandwidth of the resonators can be increased through Q loading.

The alternative to reducing resonator impedance in a single lattice, is to place multiple lattice filters in parallel. One method of doing so is to equally divide the bandwidth into $2 \times \mathrm{N}$ frequencies where $N$ is the number of lattice filters in parallel. Each lattice having two frequencies, $f_{\text {series }}$, and $f_{\text {shunt }}$ (figure $2 \mathrm{a}$ ).

$$
\begin{gathered}
B W_{3 d B} \cong \frac{2 N}{2 N-1} \Delta f \\
f_{\text {series }}(k)=f_{\text {center }}+\frac{\Delta f}{2}-(k-1) \times \frac{\Delta f}{2 N-1}, \quad \text { kodd } \\
f_{\text {shunt }}(k)=f_{\text {center }}+\frac{\Delta f}{2}-(k-1) \times \frac{\Delta f}{2 N-1}, \quad \text { keven }
\end{gathered}
$$

The approximate $3 \mathrm{~dB}$ filter bandwidth is given by (3). Note $\Delta f$ is the spacing between the maximum and minimum resonator frequencies. Equations (4-5) calculate the series and shunt frequencies for each of the $N$ lattice filters. Where $k$ is an integer representing the $k^{\text {th }}$ lattice filter $(\mathrm{k}=1$ to $\mathrm{N})$, and $\mathrm{f}_{\text {center }}$ is the desired center frequency of the overall filter response. Figure 4 shows the relationship between filter shape factor and $N$. 


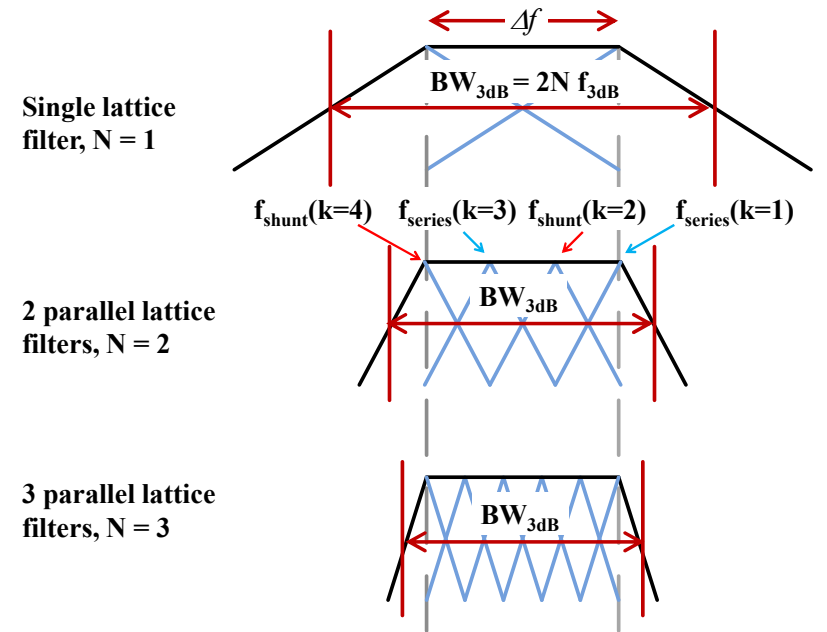

Figure 4: Conceptual diagram depicting how parallel lattice filters (parallel resonators) can be used to obtain a wide bandwidth filter response.

As the number parallel stages is increased the filter shape factor improves. This is because as $N$ increases each individual resonators loaded $Q, Q_{\text {loaded }}$, increases ( $\mathrm{f}_{3 \mathrm{~dB}}$ decreases). Therefore increasing $N$ improves filter shape factor. However, increasing $Q_{\text {loaded }}$ requires an increase in individual resonator impedance (at resonance), $R_{x}$, with respect to the termination impedance, $R_{T}$ (6). It was found through simulation that equations (6-8) yield a low ripple response. Where, $Q_{\text {unloaded }}$ is the unloaded $Q$ of the resonator and $Q_{\text {loaded }}$ is the loaded $Q$ of a single resonator with input and output terminations of $R_{T}$. From (7-8) it can be seen that the differential filter insertion loss increases with $N$.

$$
\begin{gathered}
\frac{1}{Q_{\text {loaded }}}=\frac{1}{Q_{\text {unloaded }}}\left(1+\frac{2 R_{T}}{R_{x}}\right)=\frac{\Delta f}{N f_{\text {center }}} \\
R_{x}=\frac{2 R_{T}}{\frac{\Delta f}{N} \frac{Q_{\text {unloaded }}}{f_{\text {center }}}-1} \\
I L_{\text {filter }} \cong 20 \log \left(\frac{R_{T}}{R_{x}+R_{T}}\right)
\end{gathered}
$$

Figure 5 shows the results of a model implemented in GENESYS $^{\mathrm{TM}}$ to illustrate the tradeoff between shape factor and insertion loss of the PL filter architecture. This was done for $f_{\text {center }}$ $=465 \mathrm{MHz}, \Delta f=22 \mathrm{MHz}, R_{T}=50 \Omega$ and $Q_{\text {unloaded }}=600$. The resonators frequencies were calculated using (4-5) and their impedances were calculated using (7). Also it was assumed the shunt capacitance was zero for purposes of this illustration. Hence filter insertion loss is optimistic in this example. For $N>1$ the resonators at the band edges (maximum and minimum values of $f_{\text {series }}$ and $f_{\text {shunt }}$ ) have been scaled to increase their impedance by a factor 1.5. It was found through simulation that this reduced pass band ripple. Finally as $N$ is increased ripple also increases. Ripple, due to increasing $N$, could possibly be reduced by scaling more than two resonator impedances in the filter. This added degree of freedom has not yet been investigated. Finally it should be noted that the design equations (3-8), are empirical and are not necessarily optimal.

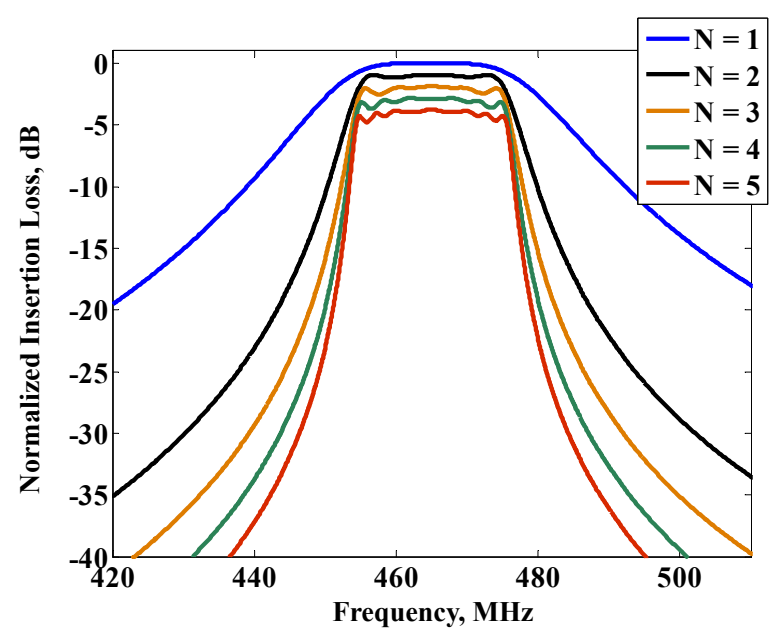

Figure 5: This illustrates the tradeoff between filter shape factor and insertion loss as $N$, filter order, is increased. All filter responses are normalized to $N=1$ case.

\section{EXAMPLE FILTER DESIGN}

Three filters were designed in this work (Filters 1-3 in tables 1-2). The first consisted of placing two $(N=2)$ basic lattice filters (figure 2a) in parallel. The second filter is derived from two parallel balun lattices (figure $3 \mathrm{a})$ and the third filter has three $(N=$ 3) differential lattice filters in parallel. In this section we will discuss the design of the third filter in greater detail. Figure 6 shows the block diagram and physical layout for the differential lattice filter design.

a) Filter schematic

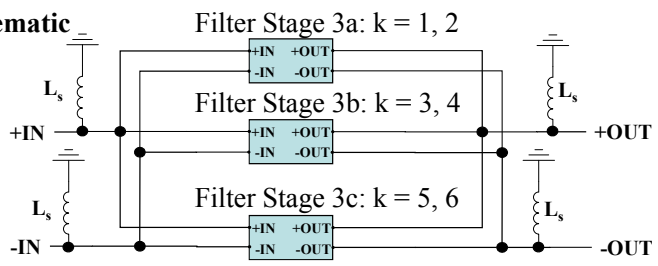

b) Physical layout

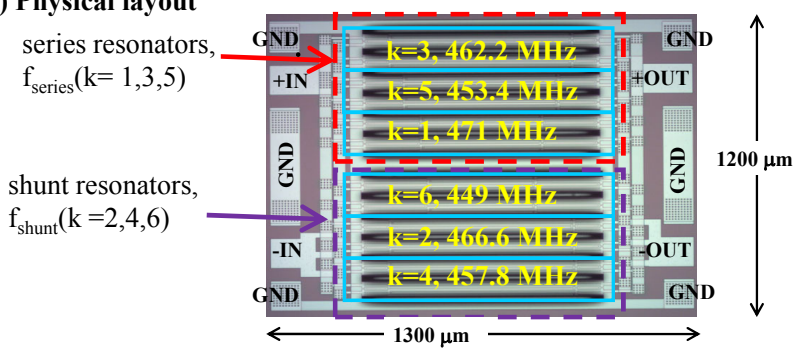

Figure 6: a) $23 \mathrm{MHz}$ parallel lattice filter schematic and b) Photograph of the filter. The blue boxes denote two identical differential resonators.

The design parameters for this filter are listed in table 1. The filter was to target an insertion loss of $-9 \mathrm{~dB}$ or better with a termination impedance of $150 \mathrm{ohms}$. Its center frequency was to be $460 \mathrm{MHz}$ with a $5 \%, 3 \mathrm{~dB}$ bandwidth $(24 \mathrm{MHz})$. The six individual resonator frequencies were calculated using equations (4-5) with $N=3, \Delta f=22 \mathrm{MHz}$, and $f_{\text {center }}=460 \mathrm{MHz}$. For this filter each single frequency differential resonator was implemented using two parallel $6^{\text {th }}$ overtone devices ( 6 electrodes). 
Table 1: Target filter parameters

\begin{tabular}{|c|c|c|c|c|c|c|c|c|}
\hline Filter \# & Filter & $\begin{array}{c}\text { Target } \\
\text { Center } \\
\text { Frequency } \\
(\mathrm{MHz})\end{array}$ & $\begin{array}{c}\text { Target } \\
3 \mathrm{~dB} \\
\text { Bandwidth } \\
(\mathrm{MHz})\end{array}$ & $\begin{array}{c}\text { Number } \\
\text { of } \\
\text { parallel } \\
\text { lattices } \\
(N)\end{array}$ & $\begin{array}{c}\text { Target } \\
\text { Insertion Loss } \\
(\mathrm{dB})\end{array}$ & $\begin{array}{c}\text { Target Ripple } \\
(\mathrm{dB})\end{array}$ & $\begin{array}{c}\text { Target } R_{T} \\
(\Omega)\end{array}$ & $\begin{array}{c}\text { Designed } \\
\Delta f(\mathrm{MHz})\end{array}$ \\
\hline \hline 1 & $\begin{array}{c}5 \mathrm{MHz} \\
\text { Lattice }\end{array}$ & 480 & 5 & 2 & -3 & $<1$ & $<200$ & 5 \\
\hline 2 & $\begin{array}{c}1.2 \mathrm{MHz} \\
\text { Balun } \\
\text { lattice }\end{array}$ & 490 & 1.2 & 2 & -9 & $<1$ & 25 \\
\hline 3 & $\begin{array}{c}23 \mathrm{MHz} \\
\text { Differential } \\
\text { Lattice }\end{array}$ & 460 & 23 & 3 & -9 & $<1.2$ & 500 \\
\hline
\end{tabular}

Table 2: Measured filter parameters

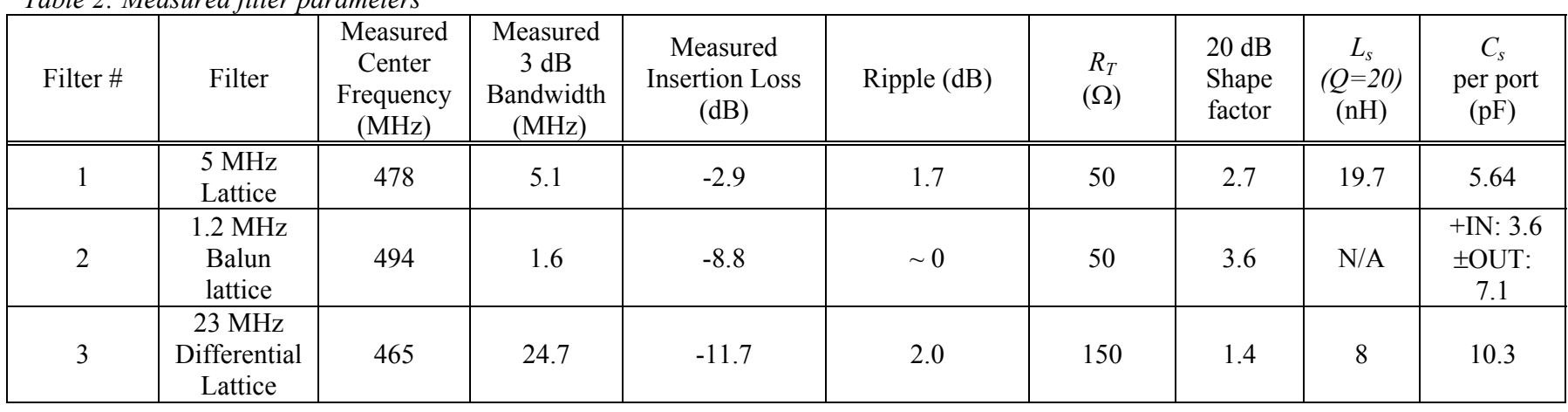

Each resonator (figure 7a) was designed to have an impedance of $2 \times R_{x}$. When placed in parallel they provide a resistance of $R_{x}$. At the time of design a differential resonator model had not yet been developed. Therefore the filter was modeled in GENESYS ${ }^{\mathrm{TM}}$ using three basic lattice filter stages in parallel (figure 6a). Filter stage $3 \mathrm{a}(\mathrm{k}=1,2)$ is the topmost filter in figure $6 \mathrm{a}$. Filter stages $3 \mathrm{~b}$ $(\mathrm{k}=3,4)$ and $3 \mathrm{c}(\mathrm{k}=5,6)$ are directly below.

\section{a) Differential resonator physical layout}

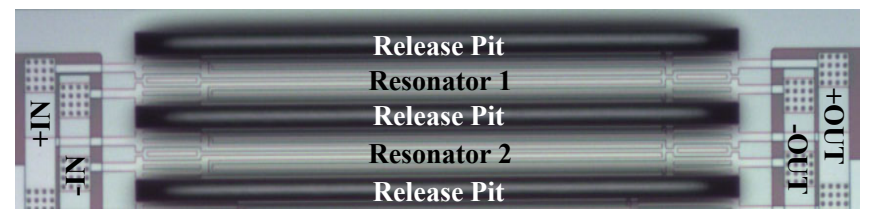

b) Diagram of differential resonator.

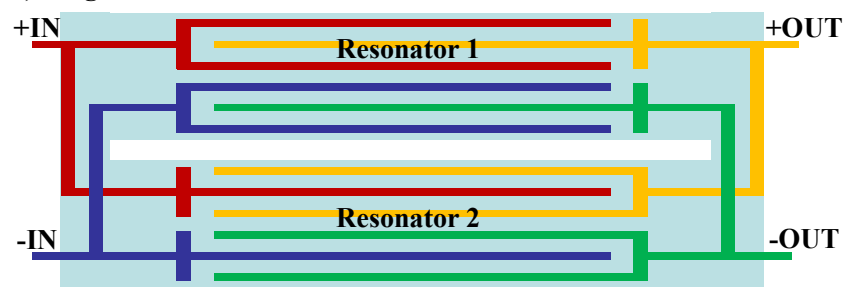

Figure 7: a) Two identical differential resonators placed in parallel. b) Mirrored layout used to match the input and output impedances of the filter.

The resonator impedance, $R_{x}$, was scaled in the RF modeling software until the desired pass band response was obtained. Note that equations (5-7) were not developed at the time however the value of $R_{x}$ (table 1 ) found through simulation agrees well with the value predicted by (7) assuming an unloaded Q between 600 and 700. In addition a shunt inductor, $L_{s}$, (figure 6a) was used to remove the shunt capacitance at the filter inputs and outputs. Since both $R_{x}$ and $C_{s}$ scale with resonator area, $L_{s}$ must be scaled as well. Ultimately IL is limited by the Q of $L_{s}$ as this dictates how well the shunt capacitance can be resonated out. Finally, to obtain resonator center frequencies in small steps of $4.4 \mathrm{MHz}$ around 460 $\mathrm{MHz}$ is difficult by changing only resonator electrode spacing. This is due to the manufacturing grid used to make the photolithographic masks for the top electrode. It sets the minimum allowed increment in the electrode spacing $(\lambda / 2)$. Hence wavelength must be an integer multiple of eight times the grid size, (9). Hence for a given grid size and material speed, $c_{m}$, all possible frequencies can be calculated using $\mathrm{f}_{\text {center }}=c_{m} / \lambda$. Where lambda is given by (9).

$$
\lambda=8 \times \text { grid size } \times M, \quad M \in \text { Integers }
$$

For a material speed of $8700 \mathrm{~m} / \mathrm{s}$, a grid size of $5 \mathrm{~nm}, \mathrm{M}=$ 472 yields a center frequency of $460.81 \mathrm{MHz}$. Incrementing or decrementing $M$ by one changes the center frequency by approximately $1 \mathrm{MHz}$. However, incrementing $M$ to obtain exactly $4.4 \mathrm{MHz}$ is not possible and the error can be quite large. As a result we have adopted a method of varying electrode width, $w_{e}$ (figure 1), for fine tuning of resonator center frequency.

\section{EXPERIMENTAL RESULTS}

As was mentioned in the previous section, several filters were designed, fabricated and tested. The target and measured filter parameters are given in tables 1 and 2. It should be pointed out that impedance matching networks at the input and output ports were used to reduce ripple for the 5 and $23 \mathrm{MHz}$ bandwidth filters. This was done in a RF simulation tool using the filters measured s- 
parameters. The matching network consisted of four shunt inductors, $L_{s}$, each having a $\mathrm{Q}$ of 20 . In addition the port impedances were increased to $150 \Omega$ for the $23 \mathrm{MHz}$ filter. Figure 8 shows the measured results (after matching in GENESYS ${ }^{\mathrm{TM}}$ ) for the three filters. The basic lattice and balun lattice models agreed well with the simulated results (dashed lines in figure 8). However, the differential lattice filter $(23 \mathrm{MHz})$ did not. It was discovered that this difference was due to a modeling oversight. The model did not capture the differential resonator. Instead, it consisted of three basic lattice filters with single input/output resonators. Hence, it did not fully capture the interaction between differential resonators. A new model with differential resonators was developed which agreed well with the $23 \mathrm{MHz}$ filter performance. Interestingly, the modeling error indicates two possible ways of improving the filters performance. First, by using the basic lattice topology instead of the differential lattice. Second, it was found (through simulation) that by balancing the number of resonator input and output electrodes for each port $(+\mathrm{IN}$, -IN, + OUT, -OUT) the differential lattice filter performance is similar to the original model (figure 8).

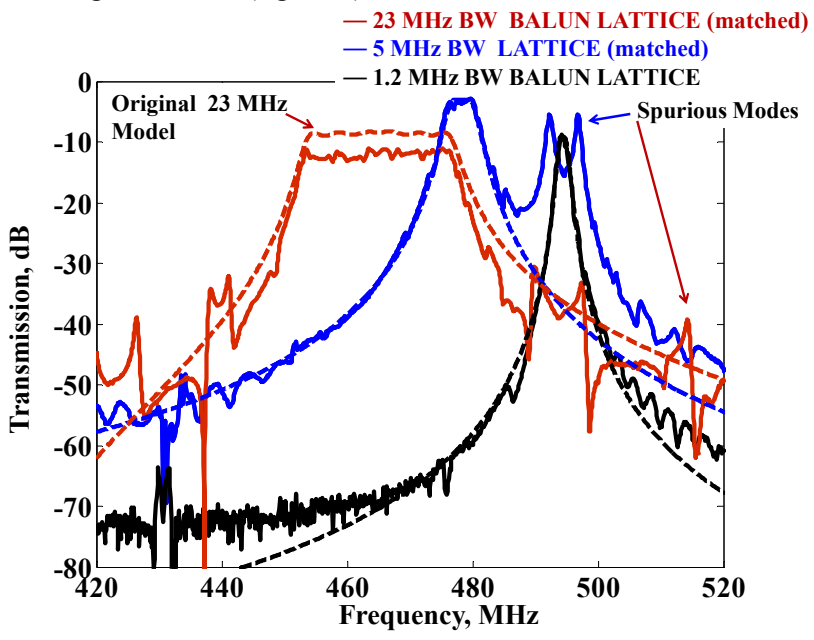

Figure 8: Measured Transmission of lattice filters. RED: $5.3 \%$ $B W$ at $463.5 \mathrm{MHz}$. BLUE: $1 \% \mathrm{BW}$ at $478 \mathrm{MHz}$. BLACK: $0.24 \%$ $B W$ at $494 \mathrm{MHz}$. Measured with AGILENT E5071C using SOLT calibration. All terminated with 50 ohms except $23 \mathrm{MHz} B W$ filter (Table 2). Dashed lines show transmission predicted by models.

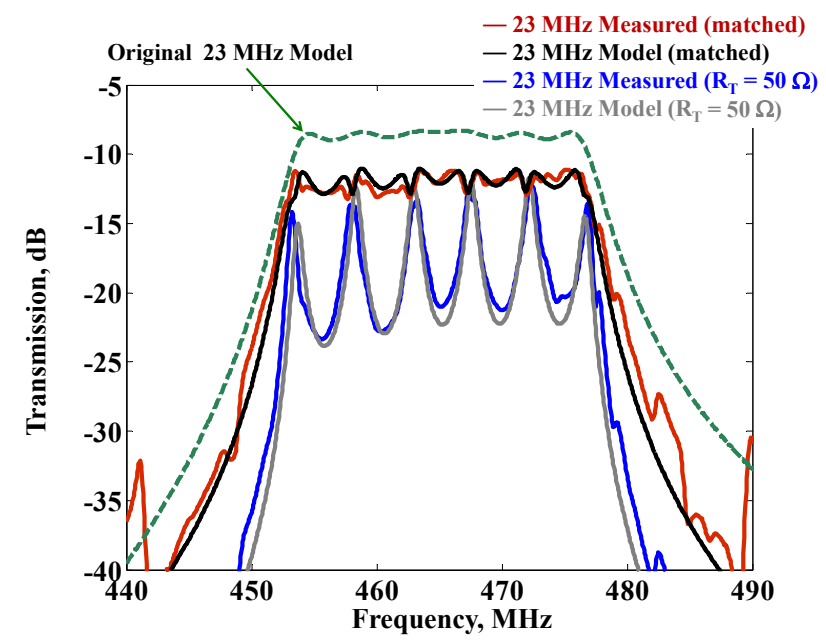

Figure 9: Comparison between original, (dashed green), and new $23 \mathrm{MHz}$ filter models, (black and grey) with fully differential resonators and measured data (red and blue).
Figure 9 shows the results of the new model. There is close agreement with the modeled and measured results once the differential resonators were fully modeled. There is a large amount of ripple in the pass band of the $23 \mathrm{MHz}$ filter when compared to the original model (figure 8 ). We believe the cause of the ripple is due to the use of unbalanced differential resonators.

Finally, the $5 \mathrm{MHz}$ basic lattice in figure 8 has very significant spurious modes in the measured transfer function. We are currently investigating methods to remove or minimize these modes. We also observed spurious modes outside the filter band of the other filters, however, they are not as severe (figure 8).

\section{CONCLUSIONS}

This work demonstrates three different filters based on the parallel lattice topology. The PL filter topology uses parallel electrically coupled lattice networks to achieve a wide bandwidth filter response. It is particularly well suited for implementation with AlN contour mode resonators as they provide key technological advantages which enable parallelization of lattice networks: small feed through capacitance, multiple frequency resonators on a single chip, and single-ended-to-differential conversion. We have demonstrated $1 \%$ bandwidth filters with insertion losses as low as $-2.9 \mathrm{~dB}$ at $500 \mathrm{MHz}$. In addition, > 5\% bandwidth filters have been achieved exceeding the $k_{t}^{2}$ limit. To the best of our knowledge this is the first demonstration of the parallel lattice filter topology.

\section{ACKNOWLEDGEMENTS}

The authors would like to thank the Microelectronics Development Laboratory staff at Sandia National Laboratories including Melanie Tuck, Jim Stevens and Craig Nakakura for AlN process development. This work was partially supported by Rockwell Collins, and we would like to acknowledge Bob Newgard, Chris Conway and Dr. Bob Potter of Rockwell Collins for that support. Sandia National Laboratories is a multiprogram laboratory operated by the Sandia Corporation, Lockheed Martin Company, for the United States Department of Energy's National Nuclear Security Administration under contract DE-AC0494AL85000.

\section{REFERENCES}

[1] M. U. Demirci, C. T.-C. Nguyen, "Single-resonator fourthorder micromechanical disk filters," 18th IEEE International Conference on Micro Electro Mechanical Systems, 2005, pp. 207-210, 2005.

[2] TriQuint data sheet for 479.5 MHz SAW filter, Part \# 855271, www.triquint.com.

[3] R. H. Olsson III, K. E. Wojciechowski, M. R. Tuck, J. E. Stevens and C. D. Nordquist, "Multi-Frequency Aluminum Nitride Micro-Filters for Advanced RF Communications," Government Microcircuit Applications and Critical Technology Conference, March 2010, In-Press.

[4] A. I. Zverev, Handbook of Filter Synthesis, John Wiley \& Sons, New York, 1967.

[5] G. Piazza, "Piezoelectric Aluminum Nitride Vibrating RF MEMS for Radio Front-End Technology", P.h.D. Thesis, Department of Electrical Engineering and Computer Science, University of California, Berkeley, 2005, p 69.

\section{CONTACT}

*K. E. Wojciechowski, tel: +1-505-284-0034;

kwojcie@sandia.gov 